\title{
Microbial Differences Between Progressive and Stable Peri-Implantitis
}

\author{
Hiroshima University \\ Shinsuke Okada \\ Hiroshima University \\ Keisuke Yasuda \\ Hiroshima University \\ Maiko Kawagoe \\ Hiroshima University Hospital \\ Mikihito Kajiya \\ Hiroshima University \\ Kazuhiro Tsuga \\ Hiroshima University
}

Yuhei Hashimoto ( $\nabla$ hash588@hiroshima-u.ac.jp )

\section{Research Article}

Keywords: Microbial, progressive, stable peri-implantitis, polymicrobial etiology, dental implant loss, bacterial flora, long-term survival rate

Posted Date: December 6th, 2021

DOI: https://doi.org/10.21203/rs.3.rs-1120921/v1

License: (c) (1) This work is licensed under a Creative Commons Attribution 4.0 International License.

Read Full License 


\section{Abstract}

Peri-implantitis has a polymicrobial etiology and is a major cause of dental implant loss. Various clinical protocols for its prevention and treatment have been proposed; however, some cases show a rapid progression with non-resolving clinical symptoms. To clear a means of differentiating between such cases, the implants with peri-implantitis in this study were categorized as the progressive group and the stable group and that two kinds of samples were obtained from the same subjects $(n=20)$. The microbiome was analyzed through pyrosequencing of the 16S rRNA gene. The stable group was found to have a diverse bacterial flora when compared to the progressive group. Porphyomonas, Fusobacterium, Treponema, Tannerella, and other periodontal pathogens were abundant in the progressive group, while Lactobacillales and Bifidobacterium were abundant in the stable group. These findings suggest that the bacterial flora dominated by these periodontal pathogens caused disease progression, while the bacterial flora dominated by Lactobacillales and Bifidobacterium stabilized the disease. Thus, the disease progression and stability of peri-implantitis may be influenced by the bacterial flora of the peri-implant pocket.

\section{Introduction}

Dental implants have a long-term survival rate in many cases ${ }^{[1-3]}$, and implant-based prosthetic replacements are a widely preferred alternative to conventional fixed or removable prostheses. However, with an increase in the function time of implants, peri-implantitis has become a major concern among $28-56 \%$ of the patients and is a leading cause of implant loss ${ }^{[4]}$. Therefore, effective prevention and management of peri-implantitis are essential in maintaining the quality of life and health of the patients. Ailing implants are often managed with antimicrobial, surgical, and local irrigation ${ }^{[5,6]}$.

Since the pathogenesis and clinical symptoms of peri-implantitis are similar to that of periodontitis ${ }^{[7]}$, its management is also based on periodontal therapy. Most patients with periodontitis respond well to treatment, and have stable periodontal tissue in the long term ${ }^{[8]}$. Similarly, over the treatment course of peri-implantitis, the progression of bone resorption may be arrested and the clinical condition may stabilize. However, in some cases the clinical conditions such as bone resorption, suppuration, and bleeding progress rapidly ${ }^{[9]}$. The reason for this existing difference is unknown. In addition, periimplantitis progresses faster than periodontitis in animal models ${ }^{[10]}$. Several studies have reported a predominance of common microbiota in these two diseases ${ }^{[11,12]}$. Studies investigating the microbiome associated with peri-implantitis using culture-independent experimental methods, such as deoxyribonucleic acid (DNA) hybridization and 16S rDNA sequencing, have revealed the predominant microbiota unique to peri-implantitis sites and those that are common between peri-implantitis and periodontitis ${ }^{[13-17]}$. Pyrosequencing of polymerase chain reaction (PCR)-amplified 16S rRNA is an innovative method of arrays that concurrently produce thousands of sequences from individual samples. This novel volume of data enables fully inclusive research of taxonomically distinct communities, revealing the microbial diversity of this disease ${ }^{[18-20]}$. 
However, these reports of bacterial flora analyses do not distinguish between cases of stable and progressive disease. Thus, it is unclear how the microbiota alters with the changes in disease progression.

In this study, we evaluated the differences in the microbiota associated with stable and progressive periimplantitis.

\section{Methods}

\section{Patients}

This study was approved by the Ethics Committee for Epidemiology of Hiroshima University (approval no. 3572) and performed in accordance with the "Ethical Guidelines for Medical Research Involving Human Subjects, Hiroshima University. A total of eight patients with either of the two kinds of peri-implantitis or both (progressive; with suppuration and stable; without suppuration) were included in this study (Figure 1). The exclusion criteria were presence of systemic diseases, antibiotic use, pregnancy, and diabetes. Informed consent was obtained from all participants.

\section{Diagnosis}

The clinical assessment of the peri-implant pocket and the presence of suppuration determined the extent of inflammation, while bone resorption was evaluated through the radiographic examination ${ }^{[21}$,

22]. Further, the extent of bone resorption was calculated by measuring the distance between the implantabutment junction and the base of the pocket. Implants of the patients with peri-implantitis showing signs of suppuration and bleeding were assigned to the progressive group, while those without the signs of suppuration and bleeding were assigned to the stable group.

\section{Sample collection}

The implants of the patients with peri-implantitis in this study were categorized as progressive group and stable group. Further, two kinds of samples were obtained from the same subjects $(n=20)$. Plaque samples from the peri-implant pockets at the greatest probing depth were obtained using a sterile paper point. Paper points were placed into sterile collection tubes and stored at $-80^{\circ} \mathrm{C}$. Following clinical examination and sample collection, the peri-implant tissues were irrigated as per the usual method.

\section{Metagenomic DNA isolation, 16S rRNA gene library preparation, and sequencing}

The paper point sample was transferred to an Eppendorf safe lock tube and processed using a PureLink ${ }^{\text {TM }}$ Microbiome DNA Purification Kit (Thermo Fisher Scientific ${ }^{\text {TM }}$, Waltham, MA, USA) according to the manufacturer's protocol. The quantity of DNA was assessed using a spectrophotometer (NanoDrop $2000^{\mathrm{TM}}$, Thermo Fisher Scientific, Waltham, MA, USA). 
The v3-v4 hypervariable regions of the bacterial 16S rRNA gene were amplified by PCR with primers (Fw: TCGTCGGCAGCGTCAGATGTGTATAAGAGACAGCCTACGGGNGGCWGCAG, Rv:

GTCTCGTGGGCTCGGAGATGTGTATAAGAGACAGGACTACHVGGGTATCTAATCC) and sequenced using an Illumina MiSeq sequencer (Illumina, San Diego, CA).

The process of DNA isolation, gene library preparation, and sequencing was performed at the $\mathrm{H}$. U. Group Research InstituteG. K.(Tokyo, Japan). The researcher and bioinformatician were blinded to the clinical information of the patients, except for their group.

\section{$16 \mathrm{~S}$ data processing}

The raw sequence data were analyzed using the QIIME2 ${ }^{[23,24]}$ (version 2021.4.0). Sequences less than 100 base pairs in length were filtered out to retain high-quality sequences. QIIME2 was used to cluster the high-quality sequences into operational taxonomic units (OTUs) with $97 \%$ similarity after trimming and filtering based on the SILVA 138.1. Singleton OTUs were removed.

\section{Statistical analyses}

Multivariate tests were performed using Permanova. The Kruskal-Wallis test was applied to compare the differences in alpha diversity of microorganisms (observed features, Simpson index, and Chao-1 index). Bray-Curtis and UniFrac distances were used for the microbial beta diversity analysis. Differences in the relative presence of the microbiome were determined using linear discriminant analysis (LDA) effect size (LEfSe) ${ }^{[25-27]}$, which can statistically extract features that can explain differences in populations of microbial communities under multiple conditions. It can be used to search for causative agents of certain phenomena.

\section{Results}

\section{Clinical information}

Nine cases of suppuration and bleeding and 11 cases without suppuration and bleeding were observed in eight patients. Bone resorption and gingival erythema were observed around all the implants. The demographic and clinical characteristics of the patients are presented in Table 1.

\section{Microbial diversity of peri-implantitis}

Two samples (Nos. 12 and 17) had insufficient DNA extraction or insufficient amplification by PCR. Nextgeneration sequencing was used for the detection, and the number of reads was the same as that of the negative control sample. The differences in the microbial diversity and composition between the progressive and stable groups were examined (Figure 2). There was no significant difference between the two groups in any of the indices. However, the progressive group had a higher Simpson index and a lower Chao1 index and observed features than the stable group. The trend was different because the Simpson index gave weightage to major species, whereas the Chao1 index gave weightage to rare species. 
According to the differences in trends among the indices, the progressive and stable groups had different microbiomes. The stable group had several rare species, while the progressive group had an even diversity of species. In the principal coordinates analysis, the progressive and stable groups formed different clusters in the ray curve distance and weighted UniFrac distance, suggesting a microbial shift from the original microbiome consisting of rare species with low pathogenicity to the inflammationinducing microbiome, as in other inflammatory diseases of the oral cavity.

\section{Taxa classification}

The taxa bar plot shows the relative frequency of the bacteria. The three most dominant bacteria of periimplantitis were Fusobacterium (uncultured bacterium) species, Porphyromonas gingivalis, and Streptococcus species. Bacteria associated with periodontal disease other than $P$. gingivalis, such as Treponema denticola, Prevotella intermedia, and Aggregatibacter actinomycetemcomitans were detected.Lactic acid bacteria, including Lactobacillus and Bifidobacterium, were rare. In addition, Lactobacillus rhamnosus, Lactobacillus fermentum, and Enterococcus faecalis, which are utilized as probiotics, were detected. The other dominant taxa are shown in Figure 3a. Streptococcus, Fusobacterium, Porphyromonas, Prevotella, Treponema, Actinomyces, Veillonella, Filifactor, Alloprevotella, Neisseria, Gemella, Tannerella, Olsenella, Campylobacter, Lactobacillus, Parvimonas, Dialister, and Eubacterium brachy groups were the most frequent at the genus level (Figure 3b). The taxa heat map showed the differences in the relative abundances between the samples (Figure 4). The results of the phylogenetic tree for each sample were similar for the same subjects. The abundance of Fusobacterium, Porphyromonas, and Streptococcus can be observed in the taxa bar plot. Lactobacillus and Bifidobacterium were more abundant in the stable group than in the progressive group.

\section{Compositional differences between the groups}

LEfSe analysis was performed to compare the relative abundance (differential abundance between the groups with LDA score $>2$ and $P<.05)$. g_Porphyomonas,g_Fusobacterium,g_Treponema, and g_Tannerella were more abundant in the progressive group, while o_Lactobacillales and g_Bifidobacterium were more abundant in the stable group. Bacteria considered as the causative pathogens of peri-implantitis were more common in the progressive group, while bacteria that were used as probiotics ${ }^{[28-31]}$ were more common in the stable group. Other LDA scores and cladograms are shown in Figure 5.

\section{Discussion}

This study explains the complex microbiome found in peri-implantitis and shows the changes in the microbiome between progressive and stable clinical condition. Furthermore, we successfully identified some representative bacteria at the species level. Though several reports compare the microbiomes of healthy implant sites with those of peri-implantitis or peri-implant mucositis ${ }^{[16,17]}$ or the microbiomes of periodontitis with those of peri-implantitis ${ }^{[14,15]}$, the results of diversity and phylogenic of microbiota 
differed widely among studies because the definition of peri-implantitis is ambiguous and because clinical conditions vary. In this study, there was no significant difference in alpha diversity, but the progressive group tended to have a higher Simpson index and a lower Chao1 index and observed features. Progressive inflammation may increase the relative abundance of certain flora and decrease the relative abundance of minor flora. Alpha diversity is the measure of the diversity within a sample. In other words, it is a sample-specific index that reflects a greater diversity of species when the values are high. The importance of "the number of different species observed" or "the equal observation of each species" differs depending on the index. Principal coordinate analysis using beta diversity showed that they formed different clusters, indicating that the samples from the stable and progressive groups show different trends within each group, although the flora composition was similar within the groups. Beta diversity is the measure of the difference in the diversity between two samples and is expressed as the distance between two points. The greater the distance, the greater the difference in the composition of both samples.

The relative frequency of periodontal disease-causing bacteria, such as $P$. gingivalis, was higher in the progressive group on the taxa bar plot, while the relative presence of lactic acid bacteria was lower in the progressive group on the taxa heat map. The "keystone pathogen" ${ }^{[32]}$ and "microbial shift" hypotheses ${ }^{[33]}$ suggest that the number of malignant bacteria can increase due to the nutrient supply to the biofilm, causing the biofilm to become more pathogenic. In the present study, P. gingivalis showed a higher relative amount in the progressive group, which is consistent with these hypotheses. Thus, changes in the peri-implant environment lead to an association between the tissue and microbiota from symbiosis to dysbiosis. The presence of common bacterial species in the progressive and stable groups and the fact that their compositional ratios are subject to microbial shifts due to environmental factors may reflect these theories. These results indicate that peri-implant inflammation is associated with changes in the bacterial flora structure. In the taxa bar plot of this study, the major bacterial taxa of peri-implant sites were partially similar to that of periodontitis. In particular, the high prevalence of $P$. gingivalis in the progressive group far exceeded our expectations.

Periodontal bacteria, such as $P$. gingivalis, $T$. denticola, Tannerella forsythia, A. actinomycetemcomitans, $P$. intermedia, Fusobacterium, Campylobacter, and other periodontal pathogens, have been reported to be associated with implant disease. P. gingivalis, T. denticola, A. actinomycetemcomitans, $P$. intermedia, Fusobacterium, and Campylobacterwere also annotated in the samples used in this study. Porphyromonas, Fusobacterium, Treponema, and Tannerella were more prevalent in the progressive group, as indicated by the LDA score. Thus, these bacteria may be the keystone species in the bacterial flora.

The level of inflammation and pocket depth should be considered when an ailing implant is identified during SPIT. If inflammation is intractable, additional treatments such as flap surgery and probiotic therapy should be considered. 
Some of the lactic acid bacteria, utilized as probiotics $[30,31,34]$, identified in this study, such as $L$. Rhamnosus. This bacterium was found in healthy children who did not have periodontal disease or tooth decay, and dietary yogurt containing L. rhamnosus L8020 significantly reduced the oral carriage of Streptococcus mutans $(P<0.01)$ and four periodontal pathogens, $P$. gingivalis, $P$. intermedia, $T$. forsythia, and Fusobacterium spp. $(P<0.01)$, whereas placebo yogurt showed no such phenomenon $(P>0.05)^{[35]}$.

In the case of peri-implantitis, these bacteria may play a role in suppressing the inflammation and can be used as probiotics. In other words, the administration of this bacterium in highly inflamed sites with periimplantitis leads to the microbiome becoming symbiotic and reduces inflammation. We aim to explore this aspect further in our future studies.

\section{Declarations}

\section{Acknowledgments}

This work was supported by the H.U. Group Research Institute G.K (Tokyo, Japan).

\section{Author contributions}

Y.H. performed the experiments, processed the data, and wrote the main part of the manuscript.

S.O. surveyed the patients, collected the samples, recommended the study design, processed the data and participated in revising the manuscript.

K.Y., and M.Kawagoe. surveyed the patients, collected the samples, and participated in revising the manuscript.

M.Kajiya., and K.T. recommended the study design and revised the manuscript.

\section{Competing interests}

The authors declare no competing interests.

\section{References}

1. Mavrogenis, A. F., Dimitriou, R., Parvizi, J. \& Babis, G. C. Biology of implant osseointegration. J. Musculoskelet. Neuronal Interact. 9, 61-71 (2009).

2. Hsu, Y. T., Lin, G. H. \& Wang, H. L. Effects of platform-switching on peri-implant soft and hard tissue outcomes: A systematic review and meta-analysis. Int. J. Oral Maxillofac. Implants 32, e9-e24 (2017).

3. Pjetursson, B. E., Asgeirsson, A. G., Zwahlen, M. \& Sailer, I. Improvements in implant dentistry over the last decade: comparison of survival and complication rates in older and newer publications. Int. J. Oral Maxillofac. Implants 29, 308-324 (2014). 
4. Zitzmann, N. U. \& Berglundh, T. Definition and prevalence of peri-implant diseases. J. Clin. Periodontol. 35, 286-291 (2008).

5. Farsai, P. S. Supportive therapy (SPT) can potentially improve implant survival rate (SR), periimplantitis, and peri-implant mucositis. J. Evid. Based Dent. Pract. 20, 101414 (2020).

6. de Waal, Y. C., Raghoebar, G. M., Meijer, H. J., Winkel, E. G. \& van Winkelhoff, A. J. Prognostic indicators for surgical peri-implantitis treatment. Clin. Oral Implants Res. 27, 1485-1491 (2016).

7. Leonhardt, A., Renvert, S. \& Dahlén, G. Microbial findings at failing implants. Clin. Oral Implants Res. 10, 339-345 (1999).

8. Lindhe, J. \& Nyman, S. Long-term maintenance of patients treated for advanced periodontal disease. J. Clin. Periodontol. 11, 504-514 (1984).

9■ Ramanauskaite, A., Becker, K. \& Schwarz, F. Clinical characteristics of peri-implant mucositis and periimplantitis. Clin. Oral Implants Res. 29, 551-556 (2018).

10. Lindhe, J., Berglundh, T., Ericsson, I., Liljenberg, B. \& Marinello, C. Experimental breakdown of periimplant and periodontal tissues. A study in the beagle dog. Clin. Oral Implants Res. 3, 9-16 (1992).

11. Shibli, J. A. et al. Composition of supra- and subgingival biofilm of subjects with healthy and diseased implants. Clin. Oral Implants Res. 19, 975-982 (2008).

12. Meffert, R. M. Periodontitis vs. peri-implantitis: the same disease? The same treatment? Crit. Rev. Oral Biol. Med. 7, 278-291 (1996).

13. Kumar, P. S., Mason, M. R., Brooker, M. R. \& O'Brien, K. Pyrosequencing reveals unique microbial signatures associated with healthy and failing dental implants. J. Clin. Periodontol. 39, 425-433 (2012).

14. Koyanagi, T. et al. Comprehensive microbiological findings in peri-implantitis and periodontitis. J. Clin. Periodontol. 40, 218-226 (2013).

15. Shiba, T. et al. Distinct interacting core taxa in co-occurrence networks enable discrimination of polymicrobial oral diseases with similar symptoms. Sci. Rep. 6, 30997 (2016).

16. Zheng, H. et al. Subgingival microbiome in patients with healthy and ailing dental implants. Sci. Rep. 5, 10948 (2015).

17. Ghensi, P. et al. Strong oral plaque microbiome signatures for dental implant diseases identified by strain-resolution metagenomics. NPJ Biofilms Microbiomes 6, 47 (2020).

18. Shchipkova, A. Y., Nagaraja, H. N. \& Kumar, P. S. Subgingival microbial profiles of smokers with periodontitis. J. Dent. Res. 89, 1247-1253 (2010). 
19. Charlson, E. S. et al. Disordered microbial communities in the upper respiratory tract of cigarette smokers. PLOS ONE 5, e15216 (2010).

20. Keijser, B. J. et al. Pyrosequencing analysis of the oral microflora of healthy adults. J. Dent. Res. 87, 1016-1020 (2008).

21. Salvi, G. E. \& Lang, N. P. Diagnostic parameters for monitoring peri-implant conditions. Int. J. Oral Maxillofac. Implants 19, 116-127 (2004).

22. Schwarz, F., Derks, J., Monje, A. \& Wang, H. L. Peri-Implantitis. J. Periodontol. 45, S246- S266 (2018).

23. Caporaso, J. G. et al. QIIME allows analysis of high-throughput community sequencing data. Nat. Methods 7, 335-336 (2010).

24. Estaki, M. et al. QIIME 2 enables comprehensive end-to-end analysis of diverse microbiome data and comparative studies with publicly available data. Curr. Protoc. Bioinformatics 70, e100 (2020).

25. Segata, N. et al. Metagenomic biomarker discovery and explanation. Genome Biol. 12, R60 (2011).

26. Fazlollahi, M. et al. The nasal microbiome in asthma. J. Allergy Clin. Immunol. 142, 834-843.e2 (2018).

27. Erawijantari, P. P. et al. Influence of gastrectomy for gastric cancer treatment on faecal microbiome and metabolome profiles. Gut 69, 1404-1415 (2020).

28. Wieërs, G. et al. How probiotics affect the microbiota. Front. Cell. Infect. Microbiol. 9, 454 (2019).

29. Ring $\varnothing$, E. et al. Probiotics, lactic acid bacteria and bacilli: interesting supplementation for aquaculture. J. Appl. Microbiol. 129, 116-136 (2020).

30. Yuki, O. et al. Effect of bovine milk fermented with Lactobacillus rhamnosus L8020 on periodontal disease in individuals with intellectual disability: a randomized clinical trial. J. Appl. Oral Sci. 27, e20180564 (2019).

31. Endo, K. et al. Comprehensive analysis of transcriptional profiles in oral epithelial-like cells stimulated with oral probiotic Lactobacillus spp. Arch. Oral Biol. 118, 104832 (2020).

32. Hajishengallis, G., Darveau, R. P. \& Curtis, M. A. The keystone-pathogen hypothesis. Nat. Rev. Microbiol. 10, 717-725 (2012).

33. Berezow, A. B. \& Darveau, R. P. Microbial shift and periodontitis. Periodontol. 2000 55, 36-47 (2011).

34. Nikawa, H. et al. Bovine milk fermented with Lactobacillus rhamnosus L8020 decreases the oral carriage of mutans streptococci and the burden of periodontal pathogens. J. Investig. Clin. Dent. 2, 187196 (2011). 
35. Maekawa, T. \& Hajishengallis, G. Topical treatment with probiotic Lactobacillus brevis CD2 inhibits experimental periodontal inflammation and bone loss. J. Periodont. Res. 49, 785-791 (2014).

\section{Tables}

Table 1 Clinical information of patients

A

\begin{tabular}{ccccccccc}
\hline Patients & A & B & C & D & E & F & G & H \\
\hline $\begin{array}{c}\text { Progressive } \\
\text { sample No. }\end{array}$ & 1 & 2 & 3 & 4 & 5 & 6,7 & 8 & 9 \\
\hline $\begin{array}{c}\text { Stable } \\
\text { sample No. }\end{array}$ & 10 & 11 & 12 & 13 & 14 & $15,16,17$ & 18 & 19,20 \\
\hline
\end{tabular}

B

\begin{tabular}{ccc}
\hline & $\begin{array}{c}\text { Progressive group } \\
\text { (sample No.1-9) }\end{array}$ & $\begin{array}{c}\text { Stable group } \\
\text { (sample No.10-20) }\end{array}$ \\
\hline Age (years) & \multicolumn{2}{c}{$69.25 \pm 9.64$} \\
Sex (\% females) & 100 & 100 \\
Probing depth (mm) & $5.9 \pm 1.7$ & $4.6 \pm 1.8$ \\
Retention type & $7 / 2$ & $9 / 2$ \\
(Screw/Cement) & & \\
Suppuration (+/-) & $9 / 0$ & $0 / 11$ \\
Bleeding on probing (+/-) & $9 / 0$ & $0 / 11$ \\
\hline Bone resorption $(\mathrm{mm})$ & $7.6 \pm 3.0$ & $4.3 \pm 3.8$
\end{tabular}

\section{Figures}

\section{Figure 1}

Samples were taken from the same oral cavity for both the progressive and stable peri-implantitis. The extent of bone resorption was calculated by measuring the distance between the implant-abutment junction and the base of the pocket. Peri-implantitis cases with suppuration and bleeding were assigned to the progressive group, while those without suppuration and bleeding were assigned to the stable group. 
A

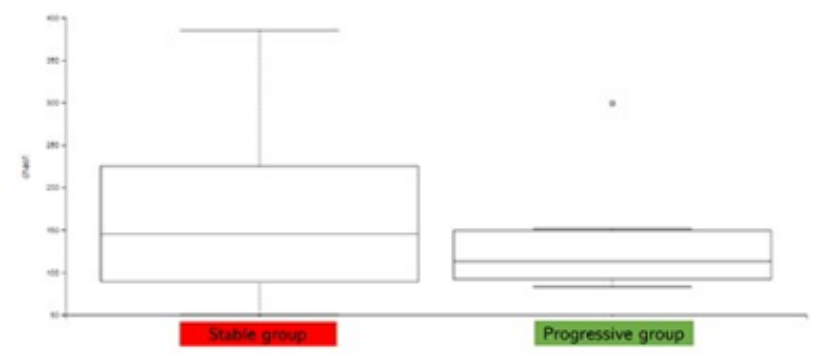

Chaol $(\mathrm{H}=0.329775, \mathrm{P}=0.565792)$

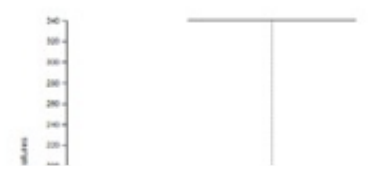

B

\section{Progressive group Stable group}

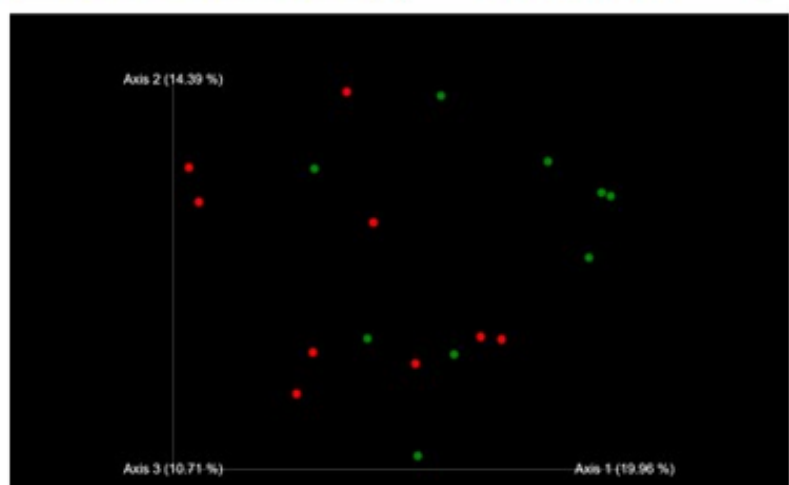

\section{Figure 2}

The oral microbiota difference between the progressive group $(n=9)$ and the stable group $(n=9)$. (A) Calculation of alpha diversity values (Chao1, observed features, and Simpson) for comparison of the total microbial diversity of peri-implantitis between the progressive group $(n=9)$ and the stable group $(n=$ 9). (B) The principal coordinate analysis charts based on Bray-Curtis and weighted UniFrac distance: comparisons between the two groups.

\section{Figure 3}

The taxa bar plot shows that the relative frequency of bacterial compositions. The three most dominant taxa of peri-implantitis are Fusobacterium (uncultured bacterium), Porphyromonas gingivalis, and genus Streptococcus. Treponema denticola, Prevotella intermedia, and Aggregatibacter actinomycetemcomitans are the bacteria associated with periodontal disease other than Porphyromonas 
gingivalis. Lactic acid bacteria, including Lactobacillus and Bifidobacterium, are rare taxa. Moreover, Lactobacillus rhamnosus, Lactobacillus fermentum, and Enterococcus faecalis which are utilized as probiotics, are detected.

\section{Figure 4}

Heat map of relative abundance of the major bacteria in the two groups by taxa. Fusobacterium, Porphyromonas, and Streptococcus show high abundance and taxa bar plot. Lactobacillus and Bifidobacterium tend be more abundant in the stable group than in the progressive group.

\section{Figure 5}

g_Porphyomonas, g_Fusobacterium, g_Treponema, and g_Tannerella are more abundant in the progressive group, whereas o_Lactobacillales and g_Bifidobacterium are more abundant in the stable group. 